\title{
Application of ultrasound technology in the study of ischemic postconditioning to protect testes from ischemia-reperfusion injury
}

\author{
E.S. Xue ${ }^{1,2}$, Q.Q. Chen ${ }^{1}$, Y. Wang ${ }^{1}$, Y. Zhang ${ }^{1,2}$, S. Chen ${ }^{1,2}$ and R.X. Liang ${ }^{1,2}$ \\ ${ }^{1}$ Department of Ultrasound, \\ The Affiliated Union Hospital of Fujian Medical University, \\ Fuzhou, Fujian, China \\ ${ }^{2}$ Institute of Ultrasound Medicine, Fuzhou, Fujian, China \\ Corresponding author: E.S. Xue \\ E-mail: enshengxue@126.com
}

Genet. Mol. Res. 13 (3): 6937-6948 (2014)

Received March 13, 2013

Accepted November 1, 2013

Published April 30, 2014

DOI http://dx.doi.org/10.4238/2014.April.30.11

\begin{abstract}
This study was designed to investigate the application of ultrasound technology in the study of ischemic postconditioning to protect testes from ischemia-reperfusion injury. Seventy-two big white rabbits were divided into mild ischemic groups (Group A: A0, A1, A2, A3), moderate ischemic groups (Group B: B0, B1, B2, B3) under ultrasound monitor, and control group $(\mathrm{N}=8)$. Groups $\mathrm{A} 0$ and $\mathrm{B} 0$ received direct perfusion, while the other groups received a different short time filling/stopped filling treatment $(15 \mathrm{~s} / 15 \mathrm{~s}, 30 \mathrm{~s} / 30 \mathrm{~s}$, or $45 \mathrm{~s} / 45$ s) three times before complete perfusion. Each group received contrastenhanced ultrasound before complete filling. At 3 days after perfusion, the testicular tissue was removed for biopsy. The parameters of testicular contrast in pre-reperfusion groups A and B differed significantly from those of their corresponding control groups $(\mathrm{P}<0.05)$. The changes in testis-related pathological indicators in groups A1 and A2 were more significant than those of group A0 $(\mathrm{P}<0.05)$, and changes in group B2 were more obvious than those of group $\mathrm{B} 0(\mathrm{P}<0.05)$. There were no
\end{abstract}


statistically significant differences in the comparison of other indicators between the corresponding groups $(\mathrm{P}>0.05)$. Ultrasound technology can help build different degree models of ischemic testes and predict the protective effect of post-ischemic treatment.

Key words: Testes; Ischemia-reperfusion injury; Time interval; Ischemic postconditioning; Ultrasonic inspection

\section{INTRODUCTION}

Testicular torsion is a urological disease that requires urgent attention. Timely restoration of blood flow is the most effective approach to save the ischemic testis (Ringdahl and Teague, 2006; Saxena et al., 2012). However, testicular torsion/restoration is a development process of ischemia-reperfusion injury, which can further aggravate the ischemic injury of the testis (Turner and Brown, 1993). The concept of ischemic postconditioning was first proposed by Zhao et al. (2003), which involves several short cycles of reperfusion/ischemia prior to the resumption of sustained blood reperfusion. This concept was confirmed to successfully reduce myocardial ischemia-reperfusion injury. Ischemic postconditioning can also relieve ischemiareperfusion injury of the testicular tissue (Shimizu et al., 2009; Zhang et al., 2011).

The ischemic degree of testes before reperfusion showed a strong influence on the effectiveness of ischemic postconditioning. As the degree of testicular ischemia increased, the effect of ischemic postconditioning was reduced, until it lost all protective value (Zhang et al., 2011). Therefore, timely and accurate assessment of the degree of testicular ischemia is important for the implementation of ischemic postconditioning. Color Doppler ultrasounds can determine the degree of testicular ischemia (Baker et al., 2000), and contrast-enhanced ultrasounds can accurately reflect the status of testicular tissue perfusion (Paltiel et al., 2006).

This study was designed to establish a model of testicular ischemia in rabbits to study the effect and mechanism of ischemic postconditioning with different time intervals on ischemia-reperfusion injury in testes with variable degrees of ischemia. In addition, the applied significance of ultrasound technology in ischemic postconditioning experiments to protect testes from ischemia-reperfusion injury was evaluated.

\section{MATERIAL AND METHODS}

\section{Subjects}

Seventy-two adult healthy rabbits were purchased from the Shanghai Sheng Wang Experimental Animal Breeding Co., Ltd. The weights of the rabbits ranged from 3.1-4.0 kg (mean, $3.41 \pm 0.44 \mathrm{~kg}$ ), and their ages ranged from 25-30 weeks (mean, $27.1 \pm 2.03$ weeks). Rabbits were randomly divided into a control group (sham operation group, $\mathrm{N}=8$ ), a mild ischemia group (A group, which included groups $\mathrm{A} 0, \mathrm{~A} 1, \mathrm{~A} 2$, and $\mathrm{A} 3, \mathrm{~N}=8$ each), and a moderate ischemia group (B group, which included groups B0, B1, B2, B3, N=8 each). Animal experiments were performed in accordance with the "Management Regulations of Laboratory Animals" and the "Implementation Rules of the Regulation of Laboratory Animal in Fujian Province", and received approval from the Affiliated Union Hospital of Fujian Medical University Ethics Committee. 


\section{Conditions of the ultrasound instrument}

Our study employed SEQUOIA-512 Color Doppler ultrasound diagnostic equipment (Siemens Company, USA) with a 3.5-inch disk image information record system and the ACQ ultrasound contrast analysis software. The II dimension frequency of the superficial probe was 13.0 MHz, the frequency of the Color Doppler was $14.0 \mathrm{MHz}$, the frequency of ultrasound contrast was $7 \mathrm{MHz}$, the span of color blood flow was $0.9 \mathrm{~cm} / \mathrm{s}$, and the machinery index and thermal index were both 0.1 . We used direct detection methods to display maximum longitudinal slices and maximum cross-sections of the testis. Color Doppler flow imaging (CDFI) and gray images of bilateral testicular sections in each group were observed and recorded before surgery and reperfusion. Then, we used the professional image analysis software (Image-Pro Plus 6) to analyze the ratio of Color Doppler flow signals in CDFI of the maximum longitudinal section of the operated side of testes to the area of the whole testes.

\section{Animal model}

This study was designed to establish a vein channel in the rabbit ear margin. Vein anesthesia $(1 \mathrm{~mL} / \mathrm{kg})$ was employed using $3 \%$ sodium pentobarbital solution, which was purchased from Shanghai West Tang Biological Limited. The rabbits were fixed in the supine position. The regional skin around the surgical point was disinfected. The animal was placed on an aseptic poriferous towel. A longitudinal line incision was applied to the scrotum root of a randomly chosen testicular side. Then, the spermatic cord structure was exposed with blunt sexual separation. The control group did not need ligation. In the ischemic groups, incomplete ligation was performed on the spermatic cord at a position $1 \mathrm{~cm}$ from the testicular upper pole under Color Doppler ultrasound monitoring. The testicular blood flow signal reduced to approximately $50 \%$ of pre-ligation levels. After ligation, the spermatic cord in the testicular lower segment showed increased tension and was purple in color, indicating obstacles to vein blood return. Then, the testis was fixed to the scrotal wall, and the perididymis and skin were sewed up to form the model of testicular blood flow obstacle. After surgery, these animals were continuously observed with dynamic ultrasound until the degree of blood deficiency changed. The ultrasound performance of mild ischemia in group A showed even echo in the testis, and the blood flow level decreased by $20-30 \%$ of pre-ligation levels. The ultrasound performance of moderate ischemia in group B showed uneven and enhanced testicular echo, and blood flow levels decreased by $5-10 \%$ of pre-ligation levels. After the changes described above appeared, the spermatic cord was exposed again. Groups A0 and B0 received recovery perfusion directly, whereas groups A1 and B1, A2 and $\mathrm{B} 2$, and $\mathrm{A} 3$ and $\mathrm{B} 3$ received filling/stopped filling three times for $15 \mathrm{~s} / 15 \mathrm{~s}, 30 \mathrm{~s} / 30 \mathrm{~s}$, and $45 \mathrm{~s} / 45 \mathrm{~s}$, respectively, as shown as Figure 1. After complete perfusion, the perididymis and skin were sewed up, and the animals were divided by group and received standard feeding.

\section{Ultrasonic contrast}

The testes received ultrasonic contrast before restoring perfusion and again 3 days after reperfusion. The ultrasonic contrast agent used was SonoVue (Italy Bracco Company). The pattern used was rapid injection of the ear vein and the dose was $0.1 \mathrm{~mL} / \mathrm{kg}$. The testicular ultrasonic contrast images were analyzed with the ACQ ultrasonic contrast software. The parameters 
included speed ( $\beta$, the speed of contrast agent flowing into the district of interest), peak time (TTP, time of the maximum signal enhanced value in the district of interest after injection of the contrast agent), strength difference of the base peak (PBD, the difference between the maximum signal and the minimum signal in the district of interest after injection of the contrast agent), and the half time of descent (DT/2, time at which the peak strength became reduced to half the PBD).

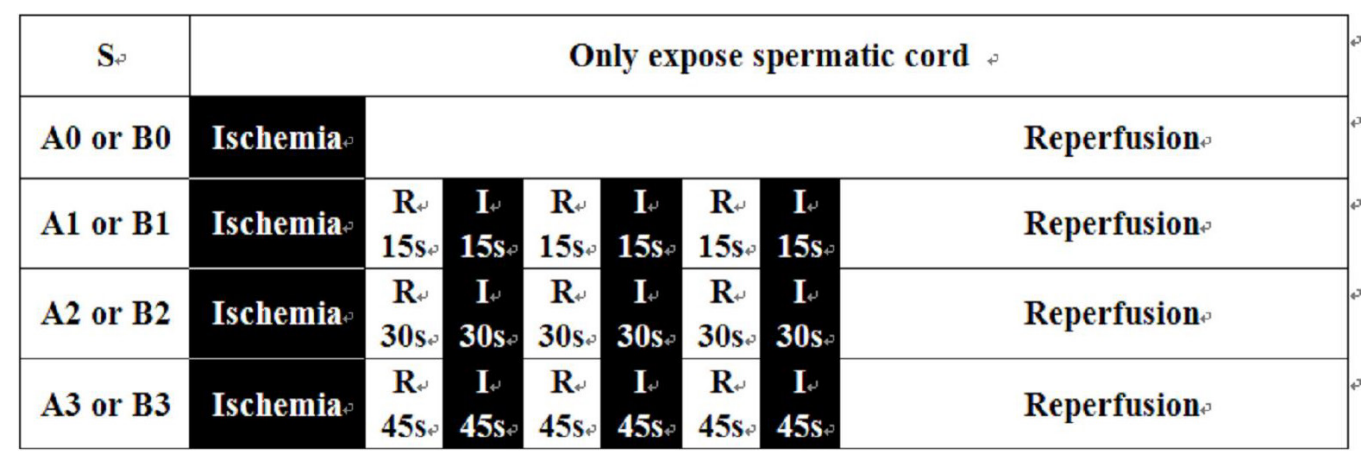

Figure 1. Schematic diagram of reperfusion/stopped perfusion at different time interval. Ischemia $=$ mild or moderate deficiency of blood; $\mathrm{R}=$ reperfusion; $\mathrm{I}=$ stopped perfusion; Reperfusion $=$ restoration of complete perfusion.

\section{Johnsen's scoring of testes and apoptosis detection of spermatogenic cells}

At the third day after cage feeding, one side of the testicular tissue was removed under anesthesia. These samples were fixed in $10 \%$ neutral formaldehyde, then dehydrated, paraffin package buried, sliced, and hematoxylin and eosin (HE)-dyed. The structure of the seminiferous tubule was observed in the optical microscope and scored according to Johnsen's scoring standard of testicular tissue biopsy (Johnsen, 1970). In situ cell apoptosis was detected by in situ end labeling using the terminal deoxynucleotidyl transferase-mediated dUTP nick-end labeling (TUNEL) method to determine the spermatogenic cell apoptosis index (AI). The apoptotic nuclei were identified by their brown color. The AI was calculated from 5 high power optical views selected in each slice, and 500 cells were counted to determine the positive cell ratio.

\section{Cysteamine asparaginase enzyme-3 (caspase-3) and Bcl-2 protein expressions detected by Western blot}

Testicular tissue proteins were extracted for gel electrophoresis, the film was transferred, dyed, bound to antibody, colored, and the image was acquired. The gray-scale value was determined using the BandScan image analysis software.

\section{Statistical analysis}

The data are reported as means $\pm \mathrm{SD}$. All data were checked for normality and variance homogeneity using the Levene test in the SPSS13.0 software package. This study was designed to apply single-factor analysis of variance to data with normal distribution and equal variance in samples, in which $\mathrm{P}<0.05$ represented statistical significance. Multiple compari- 
sons were assessed with the least significant difference (LSD) test with $\mathrm{P}<0.05$ representing a statistically significant difference.

\section{RESULTS}

\section{Testicular ultrasound contrast analysis}

In the control group, the performances of ultrasound contrasts in the bilateral testis and in the testis at the operative side were similar before and 3 days after the operation. The entire imaging process appeared as "fast forward and fast back", and the time-intensity curves almost coincided. In the ischemia groups, before reperfusion, the time-intensity curves of groups A and B appeared flat during the testicular ultrasound contrast process, and the peak values were shifted back, appearing as "slow forward and slow back". The contrast parameters and PBD of groups A and B were significantly lower than those of the control group $(\mathrm{P}<0.05)$. The TTP and DT/2 of the ischemia groups were significantly longer than those of the control group $(\mathrm{P}<0.05)$ (Table 1). Pairwise comparison of contrast parameters between subgroups within groups A and B showed no significant differences $(\mathrm{P}>0.05)$.

\begin{tabular}{|c|c|c|c|c|}
\hline$\overline{\text { Group }}$ & $\beta\left(\mathrm{x} 10^{-2}\right)$ & TTP (s) & PBD (dB) & $\mathrm{DT} / 2(\mathrm{~s})$ \\
\hline$\overline{\mathrm{S}}$ & $15.43 \pm 2.41$ & $18.69 \pm 2.52$ & $17.47 \pm 3.42$ & $11.57 \pm 1.15$ \\
\hline A0 & $11.53 \pm 1.62$ & $25.43 \pm 4.00$ & $15.15 \pm 2.38$ & $19.68 \pm 2.51$ \\
\hline $\mathrm{A} 1$ & $12.55 \pm 1.32$ & $27.15 \pm 2.89$ & $14.98 \pm 3.54$ & $20.12 \pm 3.45$ \\
\hline A2 & $13.83 \pm 1.26$ & $25.01 \pm 3.53$ & $13.34 \pm 2.18$ & $18.20 \pm 3.50$ \\
\hline A3 & $13.31 \pm 2.24$ & $28.32 \pm 5.12$ & $15.20 \pm 1.39$ & $17.56 \pm 2.53$ \\
\hline B0 & $8.03 \pm 1.39$ & $35.83 \pm 3.71$ & $8.75 \pm 0.88$ & $25.78 \pm 3.98$ \\
\hline B1 & $8.95 \pm 1.02$ & $36.98 \pm 3.85$ & $8.09 \pm 1.15$ & $26.41 \pm 5.35$ \\
\hline B2 & $9.09 \pm 1.45$ & $36.74 \pm 5.01$ & $9.50 \pm 1.17$ & $23.97 \pm 3.15$ \\
\hline B3 & $7.96 \pm 0.92$ & $37.59 \pm 2.17$ & $10.36 \pm 1.95$ & $23.48 \pm 3.51$ \\
\hline
\end{tabular}

After 3 days of reperfusion, the ultrasound contrasts of groups A and B showed a "fast forward and slow back" process. After injection of ultrasound contrast agent, the signal of the testicular envelope increased, and the testicular substance began to rapidly increase. After quickly reaching its peak intensity, the signal began to slowly fade (Figure 2). The peak values of the time-intensity curves in groups A and B significantly increased and moved forward, and all imaging parameters were significantly different in comparison with the control group ( $\mathrm{P}$ $<0.05)$. The change in group $\mathrm{B}$ was more obvious than that in group $\mathrm{A}(\mathrm{P}<0.05)$. The contrast parameters $\beta$, TTP, PBD, and DT/2 did not significantly differ between subgroups within groups $\mathrm{A}$ and $\mathrm{B}$ based on pairwise comparisons ( $\mathrm{P}>0.05$; Table 2$)$.

\begin{tabular}{|c|c|c|c|c|}
\hline Group & $\beta\left(\mathrm{x} 10^{-2}\right)$ & TTP (s) & PBD (dB) & $\mathrm{DT} / 2(\mathrm{~s})$ \\
\hline $\mathrm{S}$ & $15.01 \pm 2.10$ & $19.14 \pm 2.03$ & $16.33 \pm 2.24$ & $10.16 \pm 0.98$ \\
\hline A0 & $17.73 \pm 3.05$ & $14.15 \pm 2.13$ & $18.82 \pm 3.17$ & $13.96 \pm 1.97$ \\
\hline $\mathrm{A} 1$ & $18.12 \pm 2.24$ & $16.09 \pm 1.32$ & $21.66 \pm 2.48$ & $12.37 \pm 3.55$ \\
\hline $\mathrm{A} 2$ & $19.04 \pm 3.13$ & $15.56 \pm 1.62$ & $20.95 \pm 2.23$ & $12.63 \pm 3.85$ \\
\hline A3 & $17.94 \pm 2.87$ & $14.83 \pm 1.23$ & $21.37 \pm 2.91$ & $13.53 \pm 2.68$ \\
\hline B0 & $20.61 \pm 3.31$ & $12.74 \pm 2.69$ & $19.19 \pm 2.14$ & $12.31 \pm 2.12$ \\
\hline B1 & $23.01 \pm 3.85$ & $11.54 \pm 1.55$ & $20.36 \pm 2.41$ & $13.13 \pm 3.08$ \\
\hline B2 & $21.83 \pm 4.02$ & $11.86 \pm 3.01$ & $21.76 \pm 2.89$ & $12.96 \pm 2.13$ \\
\hline B3 & $21.34 \pm 3.77$ & $13.91 \pm 2.25$ & $20.36 \pm 2.62$ & $14.38 \pm 2.38$ \\
\hline
\end{tabular}




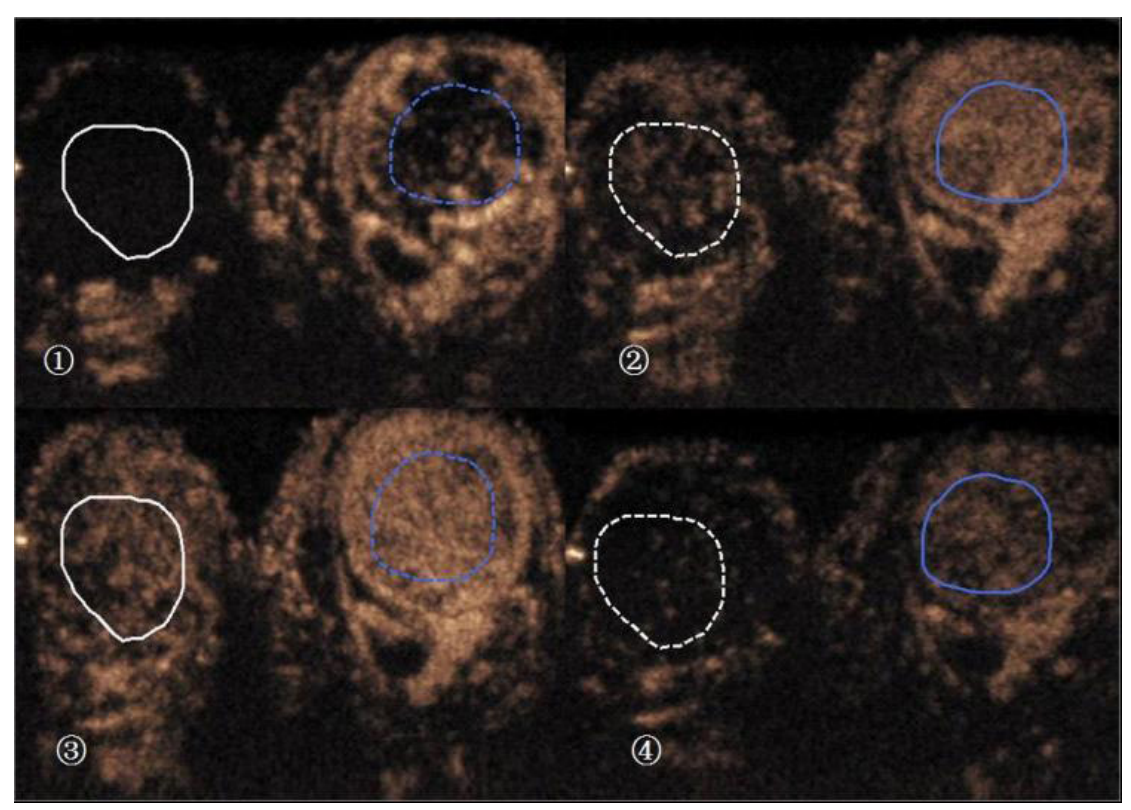

Figure 2. Testicular ultrasound contrast images in group B0 at the third day after reperfusion. 1.-4. Images in testicular operative side (blue ring) developed faster than offside, and had higher strength and extended faded time, appearing "fast forward and slow back" process.

\section{Caspase-3 and Bcl-2 protein expressions in the operative side of testicular tissue}

The ultrasound showed that the caspase- 3 content increased with increasing ischemia time and degree. The caspase- 3 contents in testes of groups A1 and A2 were significantly lower than in group A0 $(\mathrm{P}<0.05)$. Group A2 had a significantly lower caspase-3 content compared to groups $\mathrm{A} 1$ and $\mathrm{A} 3(\mathrm{P}<0.05)$, whereas there was no significant difference between groups $\mathrm{A} 0, \mathrm{~A} 1$, and $\mathrm{A} 3(\mathrm{P}>0.05)$. The caspase- 3 content in group $\mathrm{B} 2$ was significantly lower than that in group $\mathrm{B} 0(\mathrm{P}<0.05)$, whereas there was no significant difference between groups $\mathrm{B} 1, \mathrm{~B} 3$, and $\mathrm{B} 0(\mathrm{P}>0.05$; Figures 3 and 4$)$.

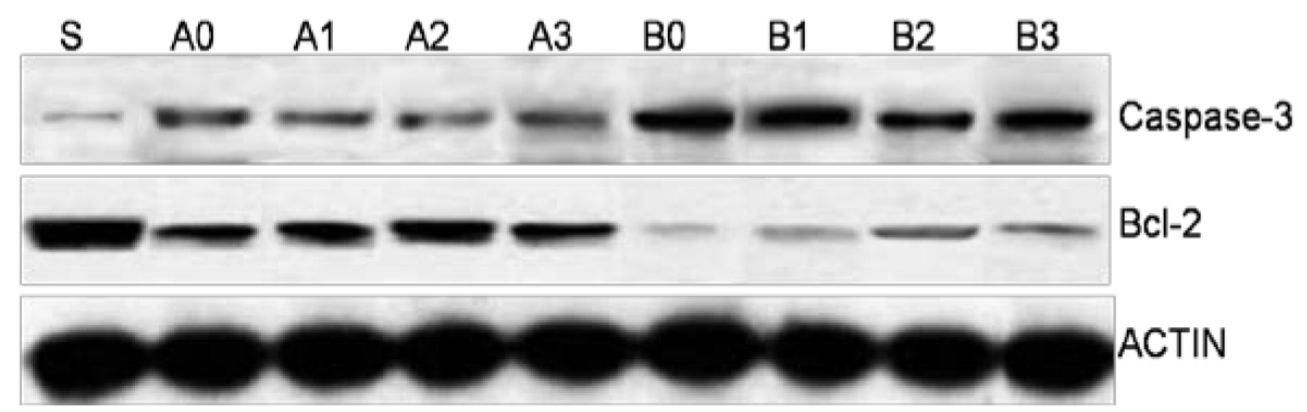

Figure 3. Caspase-3 (activated state) and Bcl-2 (activated state) expression in testicular tissue at the operative side. 


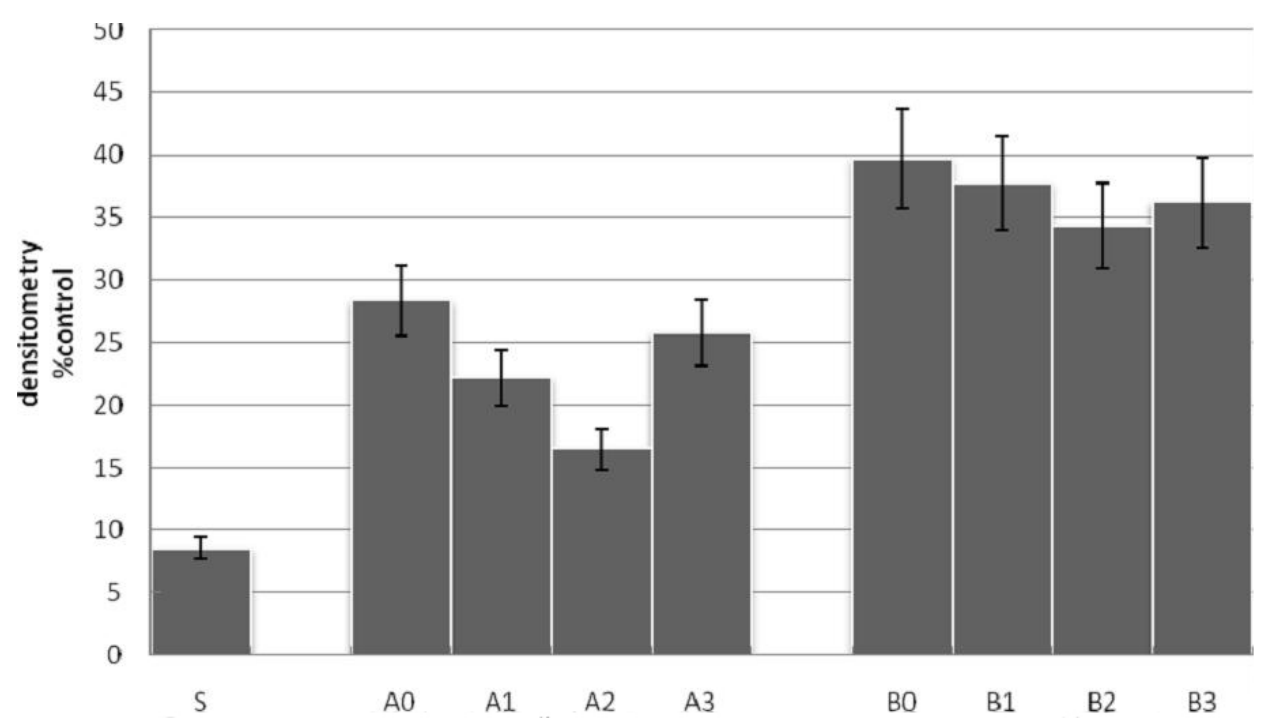

Figure 4. Histogram of caspase-3 (activated state) expression level in testicular tissue at the operative side.

The ultrasound showed that the Bcl-2 content decreased with increasing ischemia time and degree. The Bcl-2 contents in testes of groups A1 and A2 were significantly higher than those in groups A0 and A3 $(\mathrm{P}<0.05)$. Group A2 had a significantly higher $\mathrm{Bcl}-2$ content than group $\mathrm{A} 1(\mathrm{P}<0.05)$, whereas there was no significant difference between groups $\mathrm{A} 0$ and A3 $(\mathrm{P}>0.05)$. The $\mathrm{Bcl}-2$ content in group $\mathrm{B} 2$ was significantly higher than in groups $\mathrm{B} 0, \mathrm{~B} 1$, and $\mathrm{B} 3$, whereas there was no significant difference among groups $\mathrm{B} 1, \mathrm{~B} 3$, and $\mathrm{B} 0(\mathrm{P}>0.05$; Figure 5).

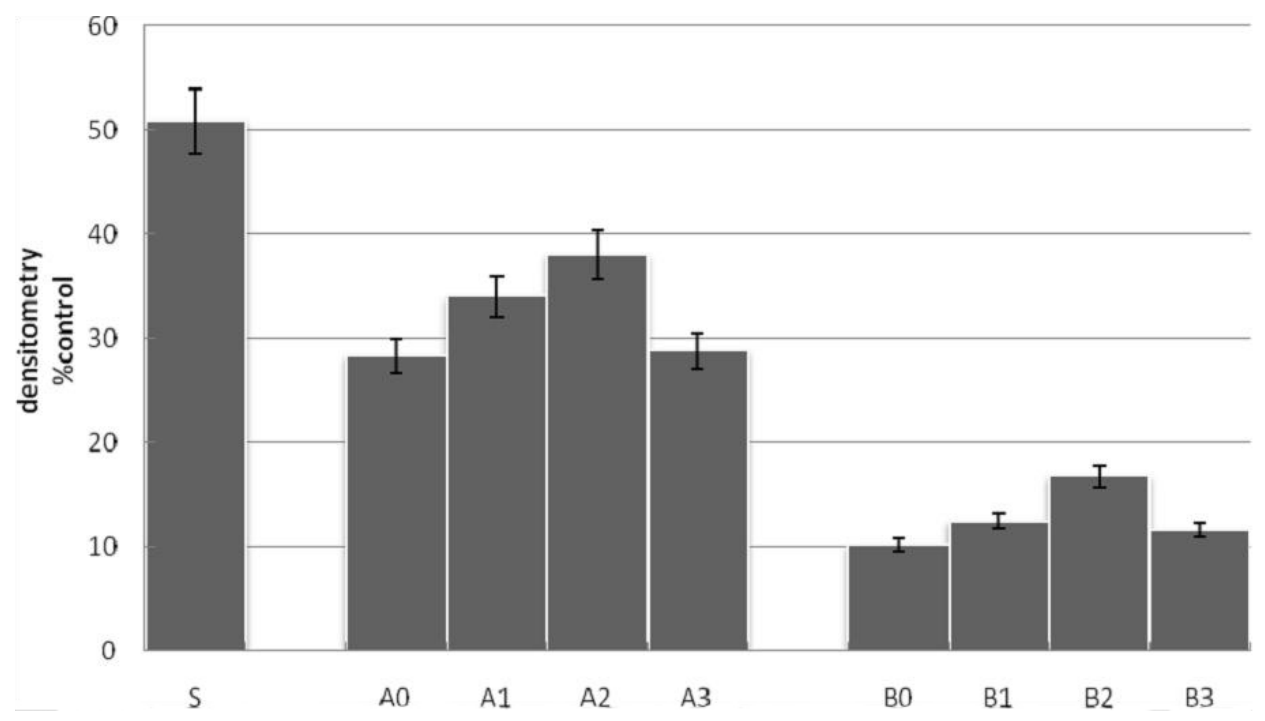

Figure 5. Histogram of Bcl-2 expression level in testicular tissue at the operative side. 


\section{Light microscope detection (HE staining)}

All levels of testicular spermatogenic cells in the control group were arranged in a neat and orderly manner. There were relatively more spermatogenic cells observed in the control group. There was no sperm at the lumen-side of seminiferous tubules. The number of spermatogenic cells at the operative side of testes gradually reduced and the Johnsen score gradually decreased $(\mathrm{P}<0.05)$ as the degree of ischemia reduced. The Johnsen score of group $\mathrm{A} 2$ was significantly higher than groups $\mathrm{A} 0$ and $\mathrm{A} 3(\mathrm{P}<0.05)$, but there was no significant difference among groups $\mathrm{A} 0, \mathrm{~A} 1$, and $\mathrm{A} 3$ or between groups $\mathrm{A} 1$ and $\mathrm{A} 2(\mathrm{P}>0.05)$. Pairwise comparisons among groups B0, B1, B2, and B3 showed no significant differences in Johnsen scores $(\mathrm{P}>0.05$; Figure 6$)$.

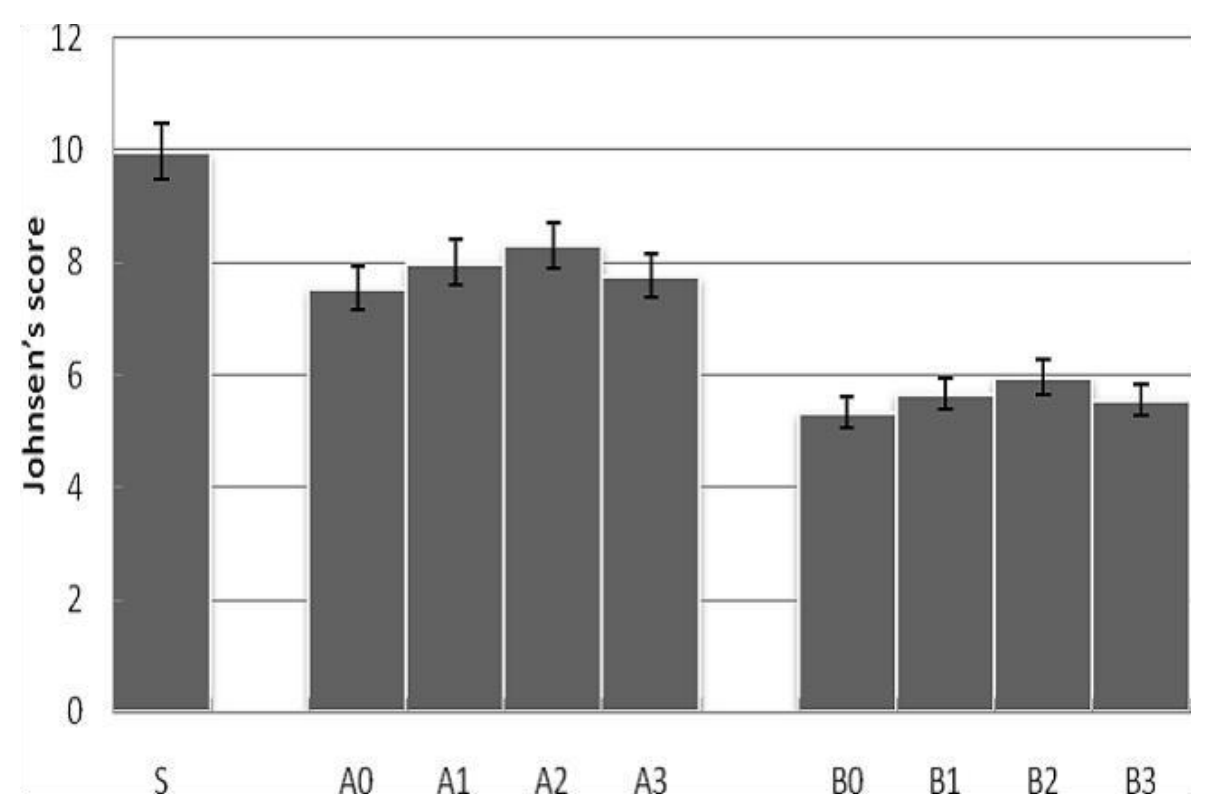

Figure 6. Histogram of Johnsen's score means in testicular tissue at the operative side.

\section{Apoptosis detection in situ}

The positive signals of TUNEL staining were located in the cell nucleus. Apoptotic cells were reduced in size, had brown-yellow nuclei, and showed nucleus condensation and chromatin gathering at the periphery. There were relatively less apoptotic cells in the control group. As the degree of ischemia increased, the number of apoptotic cells gradually increased. The apoptotic cells in the mild ischemia group were mainly primary spermatocytes with some spermatogonia. The AI values of groups A1 and A2 were significantly lower compared with the $\mathrm{A} 0$ group $(\mathrm{P}<0.05)$. There was no significant difference in $\mathrm{AI}$ between groups $\mathrm{A} 3$ and $\mathrm{A} 0$ $(\mathrm{P}>0.05)$. More apoptotic cells were observed in the moderate ischemia group. Besides large apoptotic primary spermatocytes and spermatogonia, secondary spermatocytes could also be 
observed. In the moderate ischemia group, only the AI of group B2 was significantly reduced $(\mathrm{P}<0.05$; Figures 7 and 8$)$.

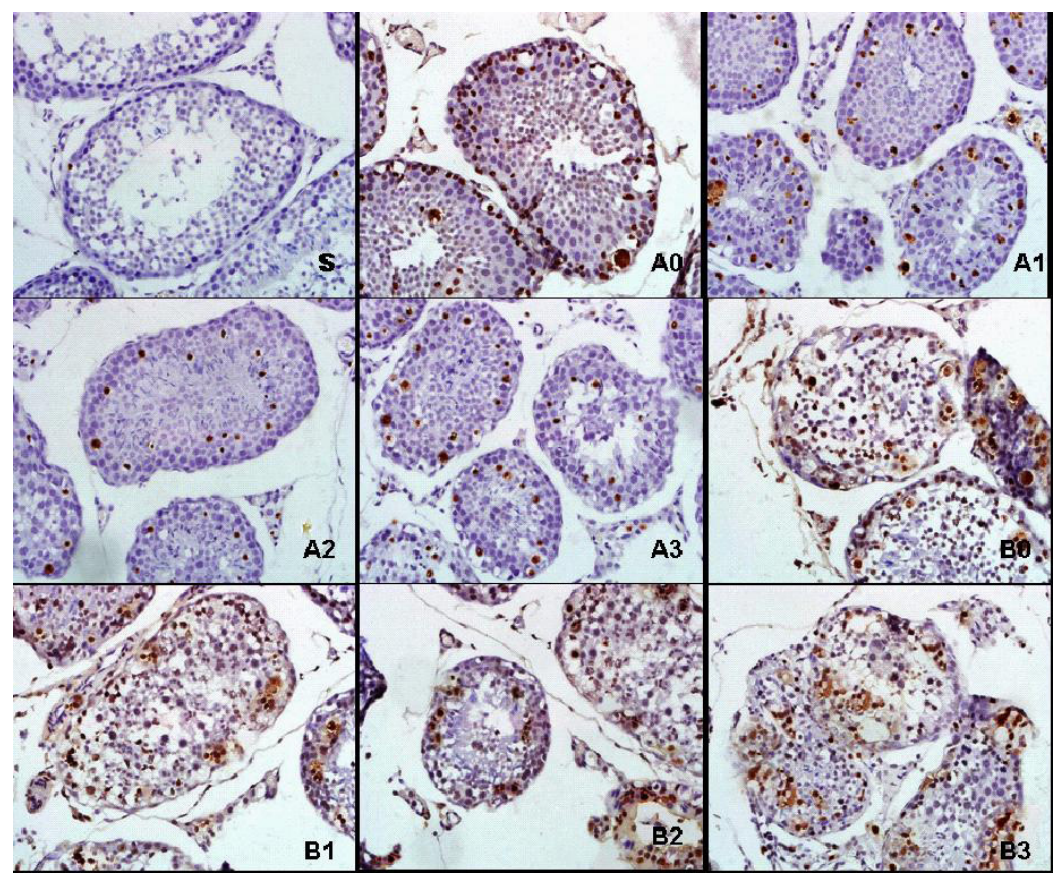

Figure 7. TUNEL staining pictures of testicular tissue at the operative side.

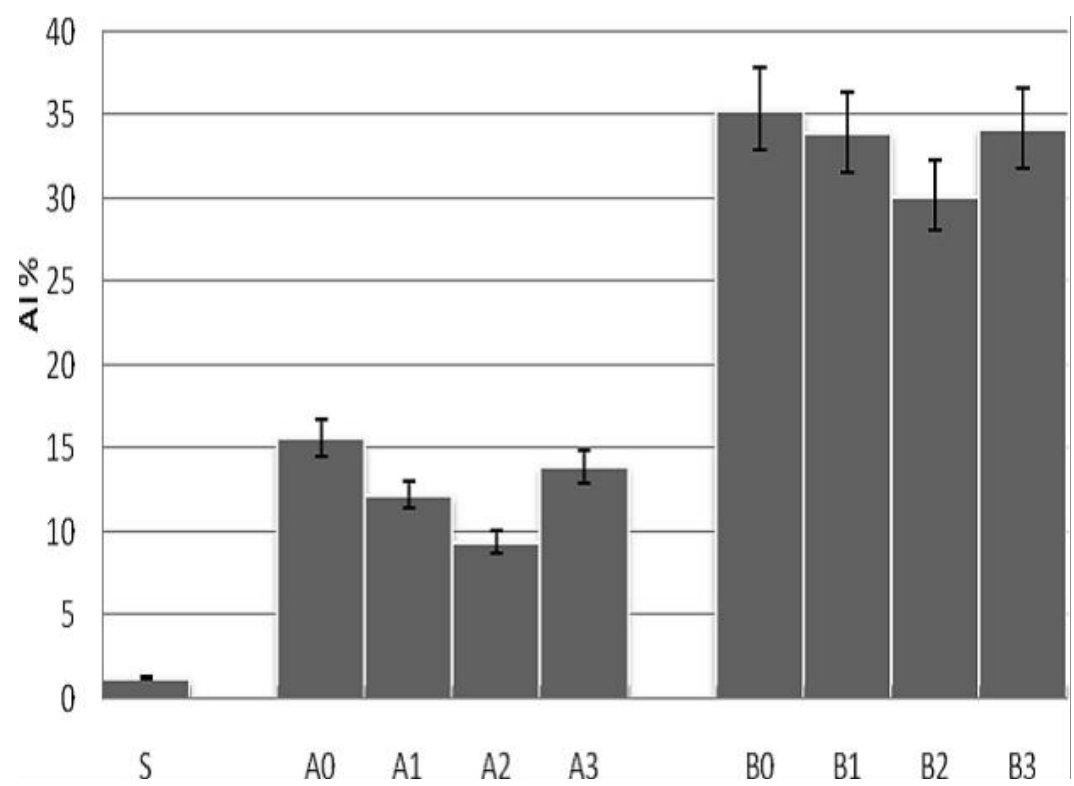

Figure 8. Histogram of apoptosis index (AI) in testicular tissue at the operative side. 


\section{DISCUSSION}

Testicular torsion can be divided into two categories: complete and incomplete reversal. The latter is more commonly observed in clinical settings. The progress of incomplete testicular torsion from the early to late stage involves a hypoxic clot followed by intravascular thrombus formation, resulting in the loss of reperfusion, which leads to ischemic necrosis in the testicular tissue. To prevent testicular torsion and ischemic necrosis, the testicular injury levels and the decision of whether or not to perform surgical restoration should be determined early. Color Doppler ultrasound can be used to visually observe the testicular size, morphology, and echo, as well as the distribution of blood flow. Contrast-enhanced ultrasounds can display the microcirculation by microbubbles in contrast agents, which can enhance the backscatter of blood cells. Therefore, the blood flow in the capillaries can be displayed with low speed, and testicular blood perfusion can be quantified (Paltiel et al., 2006; Zhang et al., 2011). Therefore, ultrasonic contrast can be used to assess the degree of testicular ischemia before ischemia reperfusion, providing an important basis to determine whether to implement ischemic postconditioning. The results of the present study showed that the echoes and blood flow distributions were similar in all groups tested. The testicular contrast time-intensity curve coincided with the contralateral curve, which confirmed that, before ischemia, the testicular microcirculation status in every group did not significantly affect the performance of the contrast-enhanced ultrasound, thereby providing a baseline curve for follow-up experiments. The experimental results showed that the contrast parameters $\beta$ and PBD of the mild ischemia groups were significantly reduced compared with those of the control group $(\mathrm{P}<0.05)$. Furthermore, TTP and DT/2 were significantly extended in the mild ischemia group, rendering an apparent "slow forward and slow back" developmental process, which indicated slow blood flow in the testis microcirculation, mild blood siltation, early testicular ischemia, and minor injury. The changes in contrast parameters in moderate ischemia groups were more obvious than those in mild ischemic groups, indicating that with the extension of ischemia, the ischemia degree increased, venous blood flow had apparent stasis, and tissue damage was aggravated. At the third day after recovery perfusion, the ultrasound contrast parameters $\beta$ and PBD of the mild ischemia groups were significantly increased, TTP was significantly shortened, and DT/2 was significantly extended compared with control groups. Furthermore, the peak value of the time-strength curve rose and moved forward. The peak value of the time-strength curve in the moderate ischemic groups also rose and moved forward, but to a greater extent, reflecting the fact that the degree of ischemia was more apparent before damage to microcirculation function, and the perfusion volume was larger after the obstruction was removed. The contrast parameters of groups A and B showed no significant differences, which indicated that functional improvement in microcirculation was not evident between ischemic postconditioning groups and direct perfusion groups.

Zhao et al. (2003) first proposed the concept of ischemic postconditioning, which involves repeated reperfusion/stopped perfusion before complete restoration of perfusion can protect a poured heart for a long time. Recently, some related experiments have confirmed that ischemic postconditioning had similar effects on ischemia-reperfusion injury in different animal tissues, including the brain, spinal cord, liver, kidney, skeletal muscle, testis, and other visceral organs. Some results have been applied to clinical settings (Staat et al., 2005; Xing et al., 2008; Chen et al., 2008; Shimizu et al., 2009; Zhao, 2010; Mewton et al., 2010; Zhang et al., 2011). The suggested protective mechanisms of ischemic postconditioning to ischemia- 
reperfusion injury of visceral organs include the inhibition of lipid peroxidation, calcium overload neutrophil activation, and activation of the remedy enzyme pathway of reperfusion injury to inhibit apoptosis (Penna et al., 2008; Abas et al., 2010; Sun et al., 2005, 2010; Granfeldt et al., 2012). Apoptosis induced by ischemia-reperfusion could be regulated by multiple proteins, and the levels of caspase-3 and Bcl-2 were shown to be determinants of cell apoptosis (Porter and Jänicke, 1999; Danial et al., 2010). Caspase-3 is one of the most important contributors to apoptosis, which once activated cannot be reversed. Bcl-2 is an important factor for inhibiting apoptosis. In the present study, the caspase- 3 content showed an increased trend with increasing degree of ischemia. In the mild ischemia groups, the caspase-3 level of the reperfusion/ stopped perfusion group at a time interval of $30 \mathrm{~s}$ significantly decreased, and this effect was greater than that observed in other time interval groups. In the moderate ischemia groups, the time interval of $30 \mathrm{~s}$ also significantly decreased the caspase- 3 content. The Bcl- 2 content showed a decreased trend with increasing degree of ischemia. In the mild ischemia groups, the $\mathrm{Bcl}-2$ level of the reperfusion/stopped perfusion group at the time interval of $30 \mathrm{~s}$ significantly increased, and the effect was greater than that of the other time interval groups. In the moderate ischemia groups, the time interval of $30 \mathrm{~s}$ also significantly increased the Bcl-2 content. These results showed that adequate ischemic postconditioning could promote the expression of the apoptotic inhibitor Bcl-2 and inhibit the activity of the apoptosis driver caspase-3, and thereby inhibit the reperfusion inducing cell apoptosis.

The protective effects of ischemic postconditioning vary among different studies under different conditions. Ischemic postconditioning could not reduce the tissue damage area when the time interval was either too short or too long, or when the cycle index was too high or too low (Kin et al., 2004; Iliodromitis et al., 2006; Manintveld et al., 2007). In the present study, in the mild ischemia groups, reperfusion/stop perfusion at a time interval of $30 \mathrm{~s}$ showed the best protective effect based on many indicators, such as Johnsen scores, the AI, and caspase-3 and Bcl-2 expression levels. However, the extended time interval of $45 \mathrm{~s} \mathrm{had}$ no significant protective effect. This may be due to the fact that increasing the time of tissue ischemia can result in increased tissue injury. Therefore, these results showed that the process of continuous reperfusion/stopped perfusion at a time interval of $30 \mathrm{~s} 3$ times had the most ideal effect on the reperfusion injury.

The protective effect of ischemic postconditioning is closely related to the degree of testicular torsion. Therefore, ischemic postconditioning could lose its protective effect on reperfusion injury under conditions of severe testicular ischemia with uneven ultrasonic echoes and disappearance of the blood flow signal (Zhang et al., 2011). The data showed that for mild ischemic testes, adequate ischemic postconditioning could enhance the Bcl-2 expression level, reduce the caspase- 3 expression levels and the number of apoptotic cells, improve the Johnsen scores, and mitigate the damage to the spermatogenic epithelium. For moderate ischemic testes, although the ischemic postconditioning at the time interval of $30 \mathrm{~s}$ could also increase Bcl-2 expression and reduce caspase- 3 expression and the number of apoptotic cells, it had no enhancing effect on the Johnsen scores. This result showed that ischemic postconditioning did not have a repair effect at all levels of spermatogenic cells and sperm. Whether it has a repair or protective effect requires further investigation. Therefore, early detection of testicular ischemia is a determining factor for prognosis. Patients with suspected testicular ischemia in a clinical context could receive timely Color Doppler ultrasonography, which, if necessary, could be combined with ultrasound contrast to observe the microcirculation state in torsional testes in order to obtain an objective assessment for determining appropriate treatment. 


\section{REFERENCES}

Abas F, Alkan T, Goren B, Taskapilioglu O, et al. (2010). Neuroprotective effects of postconditioning on lipid peroxidation and apoptosis after focal cerebral ischemia/reperfusion injury in rats. Turk. Neurosurg. 20: 1-8.

Baker LA, Sigman D, Mathews RI, Benson J, et al. (2000). An analysis of clinical outcomes using color doppler testicular ultrasound for testicular torsion. Pediatrics 105: 604-607.

Chen H, Xing B, Liu X, Zhan B, et al. (2008). Ischemic postconditioning inhibits apoptosis after renal ischemia/reperfusion injury in rat. Transpl. Int. 21: 364-371.

Danial NN, Gimenez-Cassina A and Tondera D (2010). Homeostatic functions of BCL-2 proteins beyond apoptosis. $A d v$. Exp. Med. Biol. 687: 1-32.

Granfeldt A, Jiang R, Wang NP, Mykytenko J, et al. (2012). Neutrophil inhibition contributes to cardioprotection by postconditioning. Acta Anaesthesiol. Scand. 56: 48-56.

Iliodromitis EK, Georgiadis M, Cohen MV, Downey JM, et al. (2006). Protection from post-conditioning depends on the number of short ischemic insults in anesthetized pigs. Basic Res. Cardiol. 101: 502-507.

Johnsen SG (1970). Testicular biopsy score count - a method for registration of spermatogenesis in human testes: normal values and results in 335 hypogonadal males. Hormones 1: 2-25.

Kin H, Zhao ZQ, Sun HY, Wang NP, et al. (2004). Postconditioning attenuates myocardial ischemia-reperfusion injury by inhibiting events in the early minutes of reperfusion. Cardiovasc. Res. 62: 74-85.

Manintveld OC, Te Lintel HM, Van den Bos EJ, Suurenbroek GM, et al. (2007). Cardiac effects of postconditioning depend critically on the duration of index ischemia. Am. J. Physiol. Heart Circ. Physiol. 292: H1551-H1560.

Mewton N, Ivanes F, Cour M and Ovize M (2010). Postconditioning: from experimental proof to clinical concept. Dis. Model. Mech. 3: 39-44.

Paltiel HJ, Kalish LA, Susaeta RA, Frauscher F, et al. (2006). Pulse-inversion US imaging of testicular ischemia: quantitative and qualitative analyses in a rabbit model. Radiology 239: 718-729.

Penna C, Mancardi D, Raimondo S, Geuna S, et al. (2008). The paradigm of postconditioning to protect the heart. J. Cell. Mol. Med. 12: 435-458.

Porter AG and Jänicke RU (1999). Emerging roles of caspase-3 in apoptosis. Cell Death Differ. 6: 99-104.

Ringdahl E and Teague L (2006). Testicular torsion. Am. Fam. Physician 74: 1739-1743.

Saxena AK, Castellani C, Ruttenstock EM and Hollwarth ME (2012). Testicular torsion: a 15-year single-centre clinical and histological analysis. Acta Paediatr. 101: e282-e286.

Shimizu S, Saito M, Kinoshita Y, Shomori K, et al. (2009). Ischemic preconditioning and post-conditioning to decrease testicular torsion-detorsion injury. J. Urol. 182: 1637-1643.

Staat P, Rioufol G, Piot C, Cottin Y, et al. (2005). Postconditioning the human heart. Circulation 112: 2143-2148.

Sun H, Guo T, Liu L, Yu Z, et al. (2010). Ischemic postconditioning inhibits apoptosis after acute myocardial infarction in pigs. Heart Surg. Forum 13: E305-E310.

Sun HY, Wang NP, Kerendi F, Halkos M, et al. (2005). Hypoxic postconditioning reduces cardiomyocyte loss by inhibiting ROS generation and intracellular $\mathrm{Ca}^{2+}$ overload. Am. J. Physiol. Heart Circ. Physiol. 288: 1900-1908.

Turner TT and Brown KJ (1993). Spermatic cord torsion: loss of spermatogenesis despite return of blood flow. Biol. Reprod. 49: 401-407.

Xing B, Chen H, Zhang M, Zhao D, et al. (2008). Ischemic postconditioning inhibits apoptosis after focal cerebral ischemia/reperfusion injury in the rat. Stroke 39: 2362-2369.

Zhang Y, Xue ES, Lin LW, Chen S, et al. (2011). Ischemic postconditioning reduces testis ischemia-reperfusion injury in rabbits. Zhonghua Nan Ke Xue. 17: 115-120.

Zhao ZQ (2010). Postconditioning in reperfusion injury: a status report. Cardiovasc. Drugs Ther. 24: 265-279.

Zhao ZQ, Corvera JS, Halkos ME, Kerendi F, et al. (2003). Inhibition of myocardial injury by ischemic postconditioning during reperfusion: comparison with ischemic preconditioning. Am. J. Physiol. Heart Circ. Physiol. 285: 579-588. 